\title{
Recording quality does not influence relaxation responses to lullabies; sampling criteria do, as they should
}

Reply to "Sampling criteria and recording quality influence relaxation responses to lullabies"

\author{
Mila Bertolo ${ }^{1}$, Constance Bainbridge ${ }^{2}$, Alia Martin ${ }^{3}, \&$ Samuel Mehr ${ }^{1,3,4}$ \\ ${ }^{1}$ Department of Psychology, Harvard University \\ ${ }^{2}$ Department of Communications, University of California Los Angeles \\ ${ }^{3}$ School of Psychology, Victoria University of Wellington \\ ${ }^{4}$ Data Science Initiative, Harvard University
}

The proposed Matters Arising piece (MA; https://psyarxiv.com/dcg7m) makes two claims about Bainbridge \& Bertolo et al., 2021, Nature Human Behaviour (B\&B). We reply to each claim separately.

Claim 1: The relaxation effect in $B \& B$, wherein infants' heart rates were lower during lullaby listening than non-lullaby listening, is not due to the musical content of the songs but, instead, is an artefact of lower recording quality in the non-lullabies than in the lullabies.

If infants relaxed more in response to recordings as a result of their having less noise, scratchiness, or other recording artifacts, regardless of the musical content of the songs, the results of B\&B would indeed be confounded. The MA reported signal-to-noise ratio and subjective recording quality data, showing that the lullabies used in B\&B differed from the non-lullabies on these measures. The MA did not test their effects on infants' heart rates, however; if Claim 1 is true, then higher recording quality should predict lower heart rates.

We ran such an analysis using the data from MA and B\&B. The results show that (a) neither subjective recording quality nor signal-to-noise ratio is predictive of infants' heart rates (recording quality: $r=-0.01, p=$ 0.73; signal-to-noise ratio: $r=-0.003, p=0.91$ ); and (b) the main heart rate effect holds when adjusting for recording quality and signal-to-noise ratio in a mixed-effects model (estimated difference in heart rate during lullabies vs. non-lullabies: $\beta=0.13, t=-2.81, p=0.005)$. In that model, the partial effects of stimulus features are not significantly different than zero (recording quality: $t=0.09, p=0.93$; signal-to-noise ratio: $t=0.20, p=$ 0.84). Code is at https://gist.github.com/Milberto/78346f466fe7792321571bf4c0a95272.

The relaxation results in B\&B are therefore not an artefact of stimulus recording quality. Instead, they are attributable to the musical features associated with infant-directed singing, as argued in B\&B. This is also reflected by the significant association between the degree of the songs' infant-directed style and the size of the infants' reduction in heart rate while listening to them; see "Relation between songs' infant-directedness and relaxation effects", B\&B p. 260.

Claim 2: The main effects are more likely to be general to soothing features of music and are not specific to lullabies. By selecting lullabies that were maximally "soothing" and non-lullabies that were minimally "soothing", a bias is introduced favouring a relaxation response.

This claim is consistent with our claims in B\&B. We did not argue that the relaxation effects we found were specific to lullabies. In fact, we suggested the opposite in the Discussion, arguing that the soothing features of lullabies may reflect general properties of music, including responses in other populations, like adults (see B\&B p. 261, last paragraph of left column, among others). 
With respect to the stimulus selection, worldwide, lullabies in fact differ from non-lullabies on the degree to which they are perceived as "definitely used to soothe a baby". This fact has been demonstrated in a cross-culturally balanced pseudorandom sample of four song types across multiple listener populations: MTurkers (Mehr \& Singh et al. 2018); a massive samples of adults (Mehr et al. 2019); children (Hilton \& Crowley de Thierry et al. 2021); a sample of parents of infants (in B\&B); and in non-English languages, with participants in 45 industrialized nations and 3 small-scale societies (Yurdum et al., unpublished manuscript).

Given these substantial differences in musical content across the song forms, selecting as stimuli lullabies and non-lullabies that had similar degrees of "soothingness", as the MA suggests, would amount to selecting non-representative samples of lullabies and non-lullabies. The selection method we chose avoids this issue, focusing instead on songs that are exemplars of their category (i.e., those that are perceived reliably, across cultures, to be lullabies or non-lullabies).

\section{References}

Bainbridge, C. M., Bertolo, M., Youngers, J., Atwood, S., Yurdum, L., Simson, J., Lopez, K., Xing, F., Martin, A., \& Mehr, S. A. (2021). Infants relax in response to unfamiliar foreign lullabies. Nature Human Behaviour, 5 , 256-264.

Hilton, C. B., Crowley-de Thierry, L., Yan, R., Martin, A, \& Mehr, S. A. Children infer the behavioral contexts of unfamiliar foreign songs. PsyArXiv. https://doi.org/10.31234/osf.io/rz6qn.

Mehr, S. A., Singh, M., Knox, D., Ketter, D. M., Pickens-Jones, D., Atwood, S., Lucas, C., Egner, A., Jacoby, N., Hopkins, E. J., Howard, R. M., Hartshorne, J. K., Jennings, M. V., Simson, J., Bainbridge, C. M., Pinker, S., O’Donnell, T. J., Krasnow, M. M., \& Glowacki, L. (2019). Universality and diversity in human song. Science, 366, eaax0868:1-17.

Mehr, S. A., Singh, M., York, H. W., Glowacki, L., \& Krasnow. M. M. (2018). Form and function in human song. Current Biology, 28(3), 356-368.e5. 